\title{
LONG-TERM BLADDER DYSFUNCTION AND RENAL FUNCTION IN BOYS WITH POSTERIOR URETHRAL VALVES BASED ON URODYNAMIC FINDINGS
}

\author{
MAZEN A. GHANEM, KATJA P. WOLFFENBUTTEL, ANN DE VYLDER AND RIEN J. M. NIJMAN*† \\ From the Departments of Pediatric Urology, Sophia Children's Hospital Erasmus MC, University Medical Center, Rotterdam and \\ University Hospital (AD, RJMN), Groningen, The Netherlands, and Urology Department, Pediatric Urology Section, Menoufiya \\ University (MAG), Menoufiya, Egypt
}

\section{ABSTRACT}

Purpose: Posterior urethral valves are the most common cause of congenital obstructive uropathy leading to renal failure in childhood. We investigate the influence of bladder dysfunction on renal function impairment.

Materials and Methods: We retrospectively reviewed the records of 116 patients with posterior urethral valves. After valve ablation urodynamic studies were performed in all patients. The presence of vesicoureteral reflux (VUR), renal dysplasia, serum creatinine during followup as well as urodynamic abnormalities were recorded. Mean followup was 10.3 years after valve ablation.

Results: All patients underwent endoscopic valve ablation and urinary diversion was performed in 32. Renal dysplasia was found in 9 patients. Renal function impairment at the end of followup was present in 35 patients. Urodynamic studies showed poor compliance in 30 boys, detrusor overactivity in 44, and poor compliance and detrusor overactivity in 17 . Bilateral VUR was found in 17 boys at the time of diagnosis. Urodynamic studies were normal in 23 (20\%) patients, of whom 4 had renal failure. Univariate analysis showed that poor compliance and detrusor overactivity had a significant correlation to renal function impairment in addition to bilateral VUR and renal dysplasia. In a multivariate analysis bilateral VUR was an independent prognostic factor for poor prognosis.

Conclusions: Bladder dysfunction may contribute to renal function impairment eventually but normal urodynamic findings do not preclude renal deterioration. It is likely that loss of compliance and detrusor overactivity would actually result in a valve bladder reaching its end point function.

\section{KEY WoRDS: urethra, prognosis, urodynamics}

Posterior urethral valves (PUV) are the most common form of congenital urethral obstruction with an incidence ranging from 1/3,000 and 1/8,000 male births. ${ }^{1}$ The overall prognosis of patients with PUV has improved in recent years. ${ }^{2} \mathrm{~A}$ main long-term problem in boys with PUV is impairment of renal function, for which several criteria have been identified as predictors. ${ }^{3-7}$ Subsequent series emphasized the association of voiding dysfunction with late onset renal failure. ${ }^{3}$ The cause of this late deterioration is not known, but it has been suggested to be due to bladder dysfunction reported in $75 \%$ of boys with PUV after valve ablation. ${ }^{2,8}$ However, to our knowledge few authors have reported on followup urodynamic studies in these patients and to what extent the abnormal bladder function affects the overall clinical course. We assessed the factors responsible for the outcome of renal function with special reference to the urodynamic parameters.

\section{PATIENTS AND METHODS}

The records of 116 boys with posterior urethral valves treated at the Sophia Children's Hospital were studied retrospectively. The majority of patients presented with urinary

Accepted for publication January 2, 2004.

* Correspondence: Department of Urology, Groningen University, P.O. Box 30001, 9700 RB Groningen, The Netherlands (telephone: +31503613248; FAX: +31503613043; e-mail: J.M.Nijman@chir.azg.nl).

$\dagger$ Financial interest and/or other relationship with Pfizer and Pharmacia. tract infection (UTI), abdominal swelling, urinary incontinence, septacemia or nonspecific symptoms. Infravesical obstruction on prenatal ultrasound in 17 (15\%) patients only (table 1).

Initial assessment included determination of serum creatinine, urine culture, renal ultrasonography, voiding cystourethrography and renal scintigraphy. Renal ultrasound and serum creatinine measurements were repeated at regular intervals. The diagnosis of PUV was established by voiding cystourethrography and urethroscopy. Renal dysplasia was noted in 9 patients either by imaging techniques (5) or histological examination (4, table 2).9

All patients underwent endoscopic valve ablation. At the time of valve incision or shortly thereafter temporary urinary diversion was performed in 32 patients (table 2). Urinary

TABle 1. Presenting signs and symptoms of 116 boys with PUV

\begin{tabular}{lc}
\hline & No. $(\%)$ \\
\hline Urinary tract infection & $32(28)$ \\
Prenatal presentation (ultrasound) & $17(15)$ \\
Abdominal/flank mass & $15(13)$ \\
Incontinence & $11(9.5)$ \\
Septicemia & $9(7.8)$ \\
Failure to thrive & $4(3)$ \\
Respiratory distress & $2(1.7)$ \\
Urine retention & $1(0.9)$ \\
Acute renal failure & $1(0.9)$ \\
Hematuria & $1(0.9)$ \\
\hline
\end{tabular}


TABLE 2. Results

\begin{tabular}{lc}
\hline & No. $(\%)$ \\
\hline Reflux & $52(45)$ \\
Hydronephrosis & $85(73)$ \\
Diversion & $32(28)$ \\
Dysplasia & $9(8)$ \\
Nephrectomy & $22(19)$ \\
Renal failure & $35(30)$ \\
Transplantation & $8(7)$ \\
Dialysis & $6(5)$ \\
\hline
\end{tabular}

diversion procedures consisted of high (Sober type) ureterostomies in 31 cases and vesicostomy in 1 . Severe reflux and hydronephrosis were the indication in 22 patients, while diversion was performed in 9 because of poor renal function. The Sober type of diversion immediately eliminates high intrarenal pressures with the possibility of urine passage into the bladder, providing normal bladder cycling, while at the same time urodynamic evaluation of the bladder remains possible.

Progression to chronic renal failure (CRF) was analyzed by evaluating the plasma creatinine concentrations (PCr) recorded at presentation and repeated regularly during the period of followup. Late outcome of renal function was defined by current clinical status and defined PCr as badpatient died of renal failure, had end stage renal failure (ESRF) or CRF (PCr $1.7 \mathrm{mg} / \mathrm{dl}$ or greater) or good-patient alive with $\mathrm{PCr}$ less than $1.7 \mathrm{mg} / \mathrm{dl} .3,10$ Glomerular filtration rate and inulin clearance are more reliable but these data were not available in all patients.

Urodynamic studies were performed under standard conditions, and consisted of uroflowmetry, water cystometry and pelvic floor electromyography. ${ }^{11} \mathrm{~A}$ free urinary flow was obtained if possible, followed by determination of post-void residual urine. A slow filling (5 to $10 \mathrm{ml}$ per minute) water cystometry was performed 2 or 3 times using a $7 \mathrm{Fr}$ double lumen transurethral catheter for filling and intravesical pressure monitoring during filling and voiding. Abdominal pressure was recorded using an $8 \mathrm{Fr}$ rectal feeding tube. Pelvic floor electromyography was recorded using 3 skin electrodes. In a limited number of patients suprapubic catheters were used.

The studies were done with the child in a sitting position. During filling and voiding fluoroscopy was used in all patients with monitoring of reflux, bladder neck and pelvic floor behavior during the entire procedure. Maximal cystometric bladder capacity, compliance, detrusor overactivity, relaxation of the pelvic floor muscles during voiding and residual volume were studied. To estimate normal bladder capacity we used the formula (age +2$) \times 30 \mathrm{ml}$. Urethral pressure profiles were only recorded in a small group of patients. At the time of urodynamic evaluation patient condition, complaints, renal function, presence of reflux and hydronephrosis were recorded.

Statistical analysis was performed using SPSS version 10 (SPSS Inc., Chicago, Illinois). For analysis of survival data, Kaplan-Meier curves were constructed and the log rank test was performed. Multivariate analysis was performed using Cox's proportional hazards model with $\mathrm{p}<0.05$ considered statistically significant.

\section{RESULTS}

Mean patient age \pm SD at presentation was $13.1 \pm 26$ months (range 0 to 120) and mean age at valve ablation was $14.2 \pm 28$ months (range 6 days to 164 months). Patients were followed for a mean of $10.3 \pm 6$ years (range 18 months to 22 years) after surgery. Only 3 patients were followed for less than 2 years. Unilateral nephrectomy was performed in $22(19 \%)$ patients (table 2$)$. At the end of our present followup
8 patients required renal transplantation, 6 are on dialysis and $35(30 \%)$ have ESRF (table 2).

Urodynamic bladder abnormalities were detected in 93 of 116 boys $(80 \%)$ and urodynamic findings were normal in 23 (20\%), of whom 4 had renal failure. The spectrum of urodynamic abnormalities included loss of compliance in 30 (26\%) boys, detrusor overactivity in $44(38 \%)$ and impaired pelvic floor relaxation during voiding in 31 (27\%). Bladder capacity was greater than expected (capacity for age \pm 2 SD) in 34 (29\%) boys and smaller than expected in 33. A small capacity and poorly compliant bladder was found in 14 boys (12\%), poor compliance with detrusor overactivity in $17(15 \%)$ and a small capacity with detrusor overactivity in 19 (16\%). High pressures during voiding were found in 62 boys (table 3 ). A significant post-void residual (greater than $10 \%$ of voided volume) was present in 47 boys.

At the time of urodynamic studies vesicoureteral reflux (VUR) was observed in 52 patients (unilateral 35, bilateral 17 , table 2). End stage renal disease (ESRD) developed in 8 of 17 patients with bilateral VUR $(47 \%)$ and in $10(28 \%)$ patients with unilateral VUR. Unilateral reflux with nonfunctioning of the refluxing unit occurred in 9 patients, of whom 4 had ESRD. Classification of reflux was done according to the International Reflux Study Group, ${ }^{12}$ and was grade I in 6 cases, grade II in 19, grade III in 3, grade IV in 10 and grade $\mathrm{V}$ in 12 . Of the 25 patients with high grade reflux (grades III to V) 4 presented with a normal urodynamic study.

The course of CRF and the probability of renal survival were further analyzed in relation to age at presentation, UTI, presence of renal dysplasia, urinary incontinence, VUR, prenatal diagnosis, bladder capacity, compliance and overactivity, pelvic floor relaxation, voiding detrusor pressure and residual urine. Univariate analysis, using the log rank test, showed a prognostic impact for compliance, detrusor overactivity, VUR and renal dysplasia on renal survival. Bladder capacity, incontinence, pelvic floor relaxation during voiding, amount of residual urine, high grade reflux and prenatal diagnosis had no significant influence on any variable of renal function impairment. Also, the presence of UTI at presentation was of no prognostic significance as 23 of the 32 boys with UTI had a good long-term outcome (table 4). To test whether any of these parameters had a prognostic impact, a multivariate Cox regression analysis was done including the parameters of bilateral VUR, renal dysplasia, loss of compliance and detrusor overactivity for renal survival. The presence of bilateral VUR had a hazards ratio of greater than 1 , indicating an independent marker for poor prognosis.

By combining detrusor activity and compliance 4 patient groups were considered (group 1-loss of compliance and detrusor overactivity, group 2-normal compliance and no detrusor overactivity, group 3-normal compliance with detrusor over-

TABLE 3. Urodynamic findings after valve ablation and development of ESRF

\begin{tabular}{lcr}
\hline & Total No. (\%) & No. ESRF (\%) \\
\hline Bladder capacity: & & \\
$\quad$ Small & $33(28)$ & $14(40)$ \\
Large & $34(29)$ & $3(9)$ \\
$\quad$ Normal & $49(42)$ & $18(51)$ \\
Compliance: & & \\
$\quad$ Loss of compliance & $30(26)$ & $14(40)$ \\
$\quad$ Normal & $86(74)$ & $21(60)$ \\
Bladder overactivity: & & \\
$\quad$ Detrusor overactivity & $44(38)$ & $12(34)$ \\
$\quad$ Normal detrusor & $72(62)$ & $23(66)$ \\
Pelvic floor relaxation: & $31(27)$ & $11(31)$ \\
$\quad$ Impaired & $81(70)$ & $24(69)$ \\
Good & & $6(17)$ \\
Voiding detrusor pressure: & $18(16)$ & $17(49)$ \\
$\quad$ Low & $62(53)$ & $12(34)$ \\
High & $36(31)$ & \\
Normal & &
\end{tabular}


TABLE 4. Univariate analysis of prognostic markers

\begin{tabular}{lll}
\hline \multirow{2}{*}{ Variable } & \multicolumn{2}{c}{ Outcome Parameter } \\
\cline { 2 - 3 } & Chi-Square & $\begin{array}{c}\mathrm{p} \text { V Value } \\
\text { (log rank) }\end{array}$ \\
\hline Bladder capacity & 0.9 & 0.47 \\
Bladder compliance & 4.37 & 0.04 (significant) \\
Bladder instability & 3.95 & 0.05 (significant) \\
Pelvic floor relaxation & 1.3 & 0.25 \\
Detrusor voiding pressure & 1.25 & 0.54 \\
Bladder compliance + instability & 8.15 & 0.04 (significant) \\
Residual urine & 0.21 & 0.34 \\
Urinary incontinence & 0.53 & 0.46 \\
Dysplasia & 5.81 & 0.02 (significant) \\
Urinary tract infection & 0.06 & 0.80 \\
Vesicoureteral reflux & 6.97 & 0.04 (significant) \\
High grade reflux & 1.46 & 0.23 \\
Prenatal & 1.56 & 0.22 \\
Age at presentation & 0.22 & 0.64 \\
\hline
\end{tabular}

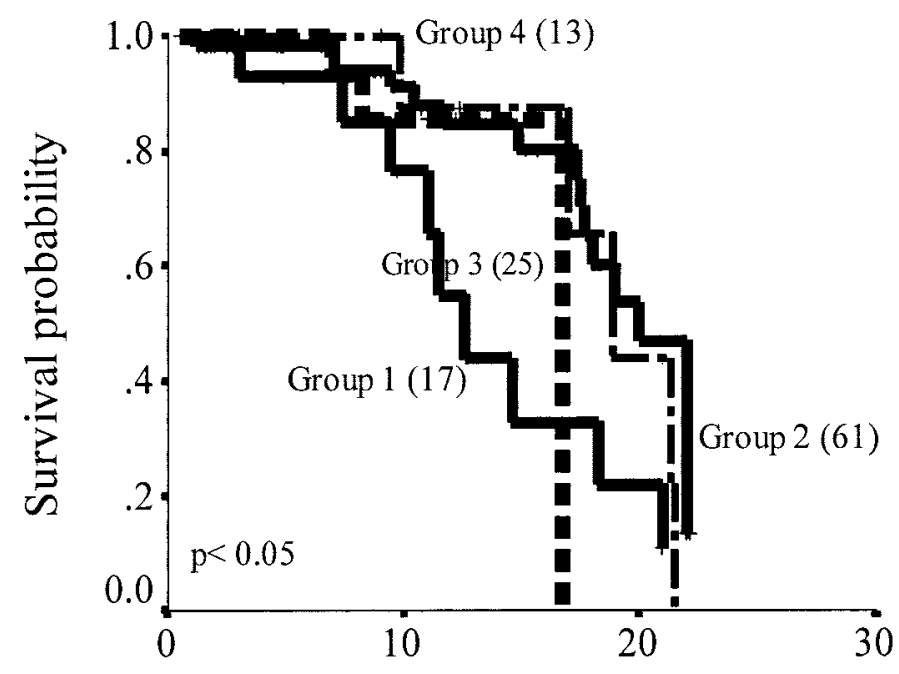

\section{Time (years)}

Kaplan-Meier curves show relationship between renal survival and combination of bladder instability and compliance. Censored patients are indicated by tic marks along line and number of patients per group is between brackets.

activity and group 4-loss of compliance without detrusor overactivity). The Kaplan-Meier curves in the figure show the prognostic influence of compliance and detrusor overactivity on time to progression to ESRD. Clearly, patients with loss of compliance and detrusor overactivity had a statistically significant poor prognosis compared to the other groups (table 4 and figure). Generally, the data on the figure are consistent with the notion that patients with normal bladder compliance and bladder contraction do better than those with loss of bladder compliance and detrusor overactivity. Loss of compliance and detrusor overactivity were simultaneously present in $17(15 \%)$ patients of whom 9 (53\%) had ESRD.

\section{DISCUSSION}

While major improvements have been achieved in lowering the mortality of patients with PUV, renal functional impairment remains a significant problem. ${ }^{6}$ Impairment of renal function, although mild at a young age, may progress to end stage renal disease. ${ }^{3}$ A considerable percentage (from $24 \%$ to $33 \%$ ) of boys with PUV will have end stage renal disease. ${ }^{13}$

Previous studies on the risk factors for developing impaired renal function in boys with PUV have been linked to patient age at presentation, prenatal diagnosis, renal dyspla- sia, initial renal function, VUR, upper tract obstruction and urinary tract infections. ${ }^{3,4,14,15}$ In addition, the type and timing of surgical interventions, duration of followup and presence of urinary continence at the age of 5 years have been described as prognostic factors. ${ }^{3}$ However, controversy exists regarding optimization of ultimate renal function in infants treated for PUV. Thus, it remains difficult to predict accurately from the initial evaluation which patients will have an unfavorable clinical outcome.

The role of bladder dysfunction in the development of renal damage is unknown. The association between incontinence due to bladder dysfunction and significant renal function impairment is an extremely important finding that is just beginning to receive attention. Parkhouse et al found that following valve ablation $45 \%$ of 5 -year-old boys had daytime urinary incontinence including $46 \%$ with progression to ESRD or CRF. ${ }^{2}$ In contrast, only $4 \%$ of continent patients with PUV at the age 5 years had a poor renal outcome on followup. ${ }^{2,3}$ In our study $36 \%$ of patients with urinary incontinence had ESRD but it was not a prognostic factor on univariate analysis.

In our patients we correlated renal deterioration to a specific urodynamic pattern, in addition to other parameters. Loss of compliance unfavorably correlated with renal function, as about $40 \%$ of patients with poor compliance had ESRD. The proportional increase of the thicker type I collagen fibers may account for decreases in compliance found in some valve bladders. Also, disorientated connective tissue seems to seriously impair the contractile and elastic characteristics of the obstructed bladder. ${ }^{16}$ Apart from reduced compliance, detrusor overactivity is associated with an increased risk of progression to ESRD. ${ }^{17,18}$ Interestingly, normal urodynamic findings did not preclude long-term deterioration.

Some reports have suggested that bladder dysfunction is important in the long-term outcome of boys with PUV and that deterioration of renal function in these patients is related to poor compliance and bladder overactivity. ${ }^{18,19} \mathrm{Lopez}$ Pereira et al reported ESRD in 22 boys of whom $89 \%$ had poor compliance and two-thirds with myogenic failure had ESRD. ${ }^{19}$ In our study the combination of detrusor overactivity and poor compliance was associated with the worst longterm prognosis for renal function as $60 \%$ of patients with this combination had ESRD.

However, the nature of kidney injury in boys with PUV is still debatable and may be due to a variety of factors. Bilateral VUR is a common cause of impaired renal function and between $50 \%$ and $70 \%$ of patients with PUV have reflux at the time of diagnosis. ${ }^{19}$ Tejani et al reported that the chance of developing CRF was 2.7 times for patients with bilateral VUR. ${ }^{3}$ The results of their study also demonstrated a poor outcome for patients with bilateral VUR, which supports the original observations of others. The favorable bladder outcome in the presence of a pop-off mechanism suggests the relationship between bladder dysfunction and VUR. ${ }^{20}$ Renal dysplasia is another important factor accounting for renal function impairment. These variable observations suggest that renal outcome after valve surgery is probably determined by a combined effect of various factors, including abnormal urodynamic findings.

\section{CONCLUSIONS}

Our study provides evidence that bladder overactivity and poor compliance have a negative influence on renal function in patients with PUV. If these results are confirmed in other studies, both factors could emerge as additional parameters for predicting the outcome for patients with PUV. Therefore, urodynamic studies appear to be helpful in determining timely intervention (medically or surgically) before it is too late. Thus, the urologist must ensure maximum recovery of bladder function to prevent progressive renal injury. How- 
ever, normal urodynamic findings do not preclude renal deterioration. Because it is impossible to predict exactly which patients will subsequently have renal failure, all with posterior urethral valves, including those with apparently normal bladders and normal initial renal function must be monitored for a long time.

\section{REFERENCES}

1. Casale, A. J.: Early ureteral surgery for posterior urethral valves. Urol Clin North Am, 17: 361, 1990

2. Parkhouse, H. F., Barratt, T. M., Dillon, M. J., Duffy, P. G., Fay, J., Ransley, P. G. et al: Long-term outcome of boys with posterior urethral valves. Br J Urol, 62: 59, 1988

3. Tejani, A., Butt, K., Glassberg, K., Price, A. and Gurumurthy, K.: Predictors of eventual end stage renal disease in children with posterior urethral valves. J Urol, 136: 857, 1986

4. Merguerian, P. A., McLorie, G. A., Churchill, B. M., McKenna, P. H. and Khoury, A. E.: Radiographic and serologic correlates of azotemia in patients with posterior urethral valves. J Urol, 148: 1499,1992

5. Warshaw, B. L., Edelbrock, H. H., Ettenger, R. B., Malekzadeh, M. H., Pennisi, A. J., Uittenbogaart, C. H. et al: Renal transplantation in children with obstructive uropathy. J Urol, 123: 737, 1980

6. Jones, D. A., Holden, D. and George, N. J. R.: Mechanism of upper tract dilatation in patients with thick walled bladders, chronic retention of urine and associated hydroureteronephrosis. J Urol, 140: 326, 1988

7. Parkhouse, H. F. and Woodhouse, C. R.: Long-term status of patients with posterior urethral valves. Urol Clin North Am, 17: 373,1990

8. Peters, C. A., Bolkier, M., Bauer, S. B., Hendren, W. H., Colodny, A. H., Mandell, J. et al: The urodynamic consequences of posterior urethral valves. J Urol, 144: 122, 1990

9. Risdon, R. A.: Renal dysplasia. I. A clinico-pathological study of 76 cases. J Clin Pathol, 24: 57, 1971

10. Denes, E. D., Barthold, J. S. and González, R.: Early prognostic value of serum creatinine levels in children with posterior urethral valves. J Urol, 157: 1441, 1997

11. Abrams, P., Cardozo, L., Fall, M., Griffiths, D., Rosier, P., Ulmsten, U. et al: The standardisation of terminology in lower urinary tract function: report from the Standardisation Subcommittee of the International Continence Society. Neurourol Urodyn, 21: 167, 2002

12. Walker, R. D.: Vesicoureteral reflux. In: Adult and Pediatric Urology. Edited by: J. Y. Gillenwater, J. T. Grayhack, S. S. Howards and J. W. Duckett. Chicago: Year Book Medical Publishers, vol. 2, pp. 1676-1708, 1987

13. Connor, J. P. and Burbige, K. A.: Long-term urinary continence and renal function in neonates with posterior urethral valves. J Urol, 144: 1209, 1990

14. Reinberg, Y., de Castano, I. and Gonzalez, R.: Influence of initial therapy on progression of renal failure and body growth in children with posterior urethral valves. J Urol, 148: 532, 1992

15. Reinberg, Y., de Castano, I. and Gonzalez, R.: Prognosis for patients with prenatally diagnosed posterior urethral valves. J Urol, 148: 125, 1992

16. Kok, D. J., Wolffenbuttel, K. P., Minekus, J. P., van Mastrigt, R. and Nijman, J. M.: Changes in bladder contractility and compliance due to urethral obstruction: a longitudinal followup of guinea pigs. J Urol, 164: 1021, 2000

17. Jaureguizar, E., Lopez Pereira, P., Martinez Urrutia, M. J., Bueno, J., Espinosa, L. and Navarro, M.: The prognosis in patients with posterior urethral valves according to the initial treatment and their urodynamic behavior. Cir Pediatr, 7: 128, 1994

18. Nijman, R. J., Scholtmeijer, R. J. and Groenewegen, A. A.: Urodynamic studies in boys with posterior urethral valves. Am Acad Pediatr, 12: 42, 1991

19. Lopez Pereira, P., Martinez Urrutia, M. J., Espinosa, L., Lobato, R., Navarro, M. and Jaureguizar, E.: Bladder dysfunction as a prognostic factor in patients with posterior urethral valves. BJU Int, 90: 308, 2002

20. Kaefer, M., Keating, M. A., Adams, M. C. and Rink, R. C.: Posterior urethral valves, pressure pop-off and bladder function. J Urol, 154: 708, 1995 